\title{
A Five Years Surveillance on Use of Antimicrobial Therapies in Biella's General Hospital (Italy): Effective Management to Reduce Antibiotic Resistance and Improve Outcome
}

\author{
Ussai $\mathbf{S}^{1,2^{*}}$, D'Aloia $F^{4}$, Lanzone $L^{3}$, Frassati $C^{4}$, Scarpetta $S^{2}$, Penna $A^{2}$ and Bonelli $\mathbf{G}^{2}$ \\ ${ }^{1}$ Bocconi School of Management, Bocconi University, 20100 Milano, Italy \\ ${ }^{2}$ Office of the Director General, Biella General Hospital, 13875 Ponderano Bl, Italy \\ ${ }^{3}$ Division of Pharmacy, Biella General Hospital, 13875 Ponderano BI, Italy \\ ${ }^{4}$ Infection Control Committee, Biella General Hospital, 13875 Ponderano BI, Italy
}

*Corresponding author: Silvia Ussai, Bocconi School of Management, Bocconi University, 20100 Milano, Italy, Tel: +393451700980; E-mail: ussai.silvia@gmail.com

Received date: March 24, 2017; Accepted date: April 10, 2017; Published date: April 17, 2017

Copyright: (c) 2017 Ussai S, et al. This is an open-access article distributed under the terms of the Creative Commons Attribution License, which permits unrestricted use, distribution, and reproduction in any medium, provided the original author and source are credited.

\section{Short Communication}

The risk of nosocomial infection due to multidrug resistance is a substantial threat in modern day treatment. According to the European Surveillance of Antimicrobial Consumption Network, systemic use of antibiotics increased to 3.4 -fold in 2015, outside the hospitals in comparison to 2014 data. However, during 2011 to 2015, systemic use of antibiotics significantly increased in the hospitals and data showed this trend varied from 1.0 to 2.9 DDD (defined daily doses) per 1000 inhabitants and per day [1-5].

In present scenario, there are no great success stories in the development of new antibiotics. Alternative therapy of antibiotics is one of the novel approaches to fight the growth of antibiotic-resistant pathogens. The alternative therapy must be applicable for both preventive and therapeutic measure, such as, modulate the gut ecosystem, either via consuming additives or faecal transplantation. It has been observed that several alternative methods enhance antibiotic efficacy by increasing bactericidal effect and reducing options for antibiotic resistance. Therefore, continue to use alternative therapy along with antibiotic to restrict the bacterial evolution for development of resistance [6].

European Medicines Agency (EMA) plays a big role in overall global response against threat of antimicrobial resistance through their continuous endeavour of supporting the development of new medicines and treatment approaches; promoting responsible use of existing antibiotics; collecting antimicrobial consumption data to guide policy and research. EMA and other international organization work together for preparing a list to indicate the member states about the harmonized use of antibiotics and restrict the misuse or over use of antibiotics. This initiative facilitates member states to supervise the use of antimicrobials in humans, food-producing animals and food, and evaluate the progress for implementing their action plans against antimicrobial resistance [7].

Since 2011, General Hospital of Biella developed its Antimicrobial Stewardship Program (ASP), executed by multidisciplinary antimicrobial utilization teams comprising of physicians, pharmacists, microbiologists, epidemiologists and infectious disease specialists, with adequate experience in their respective fields, to optimize antimicrobial therapy, minimize treatment-related cost, get better clinical outcomes and safety. To reduce or stabilize the antimicrobial resistance, it is necessary to strictly follow the existing guideline. Moreover, prudent guidelines for using of antibiotics were defined, based on Pharmacokinetic (PK) and Pharmacodynamic (PD) properties of antibiotics. Other than this, Hospital introduced several other strategies to control high antimicrobial resistance such as, advanced diagnostic testing, clinical response and effects on the microbiota.

In a 5-year surveillance on consumption and sensitivity, amoxicilinclavulanic acid has been the most consistently prescribed antibiotic inside the Hospital, on top from 2011 to 2016 with a steady number of prescriptions (between 51.000 and 55.000 prescriptions/year) during the studied period.

Due to the relatively low cost and broad spectrum of amoxicilinclavulanic acid-considered as the first line for a plenty of infections, from the airway to the urinary tract, including skin infections-Authors performed a cross analysis on its bacterial sensitivity trends, in order to assess a susceptibility rate over time.

The sensitivity data to this antibiotic proved a stable pattern for Escherichia coli, Haemophilus parainfluenzae, Klebsiella species, Proteus mirabilis and even Streptococcus pneumonia; for all of them sensitivity was above $90 \%$.

It's worthy of note that the organism Klebsiella pneumonie moved from a $72 \%$ of sensitivity in 2012 to $97 \%$ in 2016, statistically significative ( $\mathrm{p}$ value 0.03 ); this is probably due to a specific clinical policy, i.e., a better isolation of the patients exposed or an earlier detection of the microorganism.

According to the recent literature warnings, we observed an increasing tendency on resistance of Escherichia coli and Klebsiella species with extended-spectrum beta-lactamases (sensitivity less than 40\%) and Staphylococcus aureus (below 60\%).

Biella's General Hospital Governance Program demonstrated how every member of the Hospital take on responsibility for maintaining the effectiveness of antibiotics, perform their role, and finally succeeded in reducing antibiotic resistance. In summary, both "frontend" interventions: (i) The development of situation-specific treatment guideline; (ii) Education of prescribers; (iii) Accurate organismidentification; (iv) Understanding Pharmacokinetic (PK) and Pharmacodynamics (PD) properties of drugs which helps selecting optimal dose and duration of antibiotics; and "Back-end" interventions, including: (i) Clinical decision support; (ii) Postprescription review and feedback; (iii) Determination of therapy length; and (iv) Antibiotic heterogeneity (cycling and mixing) are extremely important to maintain an effective antimicrobial stewardship. 
Citation: Ussai S, D'Aloia F, Laura L, Frassati C, Scarpetta S, et al. (2017) A Five Years Surveillance on Use of Antimicrobial Therapies in Biella's General Hospital (Italy): Effective Management to Reduce Antibiotic Resistance and Improve Outcome. J Pharmacovigil 5: 227. doi:10.4172/2329-6887.1000227

Page 2 of 2

\section{References}

1. http://ecdc.europa.eu/en/eaad/antibiotics-get-informed/antibioticsresistance-consumption/Documents/antibiotics-EARS-Netsummary-2016.pdf

2. Baggs J, Fridkin SK, Pollack LA, Srinivasan A, Jernigan JA (2016) Estimating national trends in inpatient antibiotic use among US hospitals from 2006 to 2012. JAMA Int Med 176: 1639-1648.

3. Dumartin C, L'hériteau F, Péfau M, Bertrand X, Jarno P, et al. (2010) Antibiotic use in 530 French hospitals: results from a surveillance network at hospital and ward levels in 2007. J Antimicrob Chemother 65: 2028-2036.
4. Plüss-Suard C, Pannatier A, Zanetti G (2009) Antibiotic use in acute care hospitals: comparison of Switzerland with other European countries. JAC 58: 557-660.

5. Versporten A, Bolokhovets G, Ghazaryan L, Abilova V, Pyshnik G, et al. (2014) Antibiotic use in eastern Europe: a cross-national database study in coordination with the WHO Regional Office for Europe. Lancet Infect Dis 14: 381-387.

6. Allen HK, Trachsel J, Looft T, Casey TA (2014) Finding alternatives to antibiotics. An New York Acad Sci 1323: 91-100.

7. http://www.ema.europa.eu/ema/index.jsp?curl=pages/special_topics/ general/general_content_000439.jsp 